\title{
231 Colecistectomias Laparoscópicas em Ambulatório: Que Resultados?
}

\author{
231 Laparoscopic Cholecystectomy in Ambulatory: What Results?
}

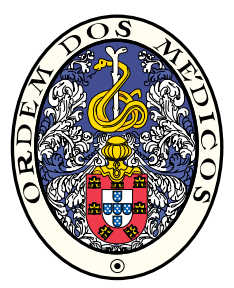

\author{
André GOULART ${ }^{1}$, Margarida DELGADO ${ }^{1}$, Maria Conceição ANTUNES ${ }^{1}$, João BRAGA DOS ANJOS ${ }^{1}$ \\ Acta Med Port 2013 Sep-Oct;26(5):564-568
}

\section{RESUMO}

Introdução: A colecistectomia laparoscópica é actualmente o gold standard no tratamento da patologia litiásica vesicular e colecistite aguda. A sua realização em regime de cirurgia de ambulatório permanece em discussão. O presente estudo tem por objectivo analisar a qualidade e segurança das colecistectomias laparoscópicas realizadas pela Unidade de Cirurgia de Ambulatório do Hospital de Braga e comparar os resultados com outros centros europeus que realizam colecistectomia laparoscópica em regime de ambulatório. Material e Métodos: Estudo observacional prospectivo de doentes submetidos a colecistectomia laparoscópica em regime de ambulatório durante 26 meses. Foram recolhidos dados referentes à demografia do doente, complicações peri e pós-operatórias, tempo cirúrgico e tempo de permanência no recobro e internamentos não programados.

Resultados: Foram submetidos a colecistectomia laparoscópica em regime de ambulatório com pernoita (alta $<24 \mathrm{~h}) 231$ doentes. Três doentes tiveram intercorrências intra-operatórias com necessidade de conversão para laparotomia e quatro doentes foram internados após a cirurgia. O tempo médio de cirurgia foi de 58 minutos e o tempo médio de recobro de 19h19m. A morbilidade pósoperatória foi de $7,8 \%$ tendo, ocorrido dois internamentos não programados.

Discussão: Uma das discussões que existe em torno da colecistectomia laparoscópica em regime de ambulatório é a necessidade de vigilância hospitalar durante a primeira noite após a cirurgia. Na nossa unidade, iniciamos a realização da colecistectomia laparoscópica em regime de ambulatório com pernoita hospitalar. Os resultados de mais de dois anos e 231 doentes operados a colecistectomia laparoscópica mostram que é uma técnica perfeitamente segura em regime de ambulatório.

Conclusões: Os dados do estudo mostram que a colecistectomia laparoscópica é uma técnica segura em regime de ambulatório, com resultados semelhantes comparativamente com outros centros europeus.

Palavras-chave: Colecistectomia Laparoscópica; Procedimentos Cirúrgicos Ambulatórios; Morbilidade.

\section{ABSTRACT}

Introduction: Laparoscopic cholecystectomy is the gold standard procedure for the treatment of lithiasic gallblader pathology and acute cholecystitis. Whether or not it should be done as ambulatory surgery is still being discussed. The present study aims to analyze the quality and safety of laparoscopic cholecystectomy conducted by the Ambulatory Unit of Hospital de Braga and compare the results with those from other European surgical centers performing LC as ambulatory surgery.

Material and Methods: Observational prospective study of patients submitted to laparoscopic cholecystectomy in ambulatory surgery during a period of 26 months. Data regarding patients' demography, peri- and postoperative complications, surgical time, time in recovery room, and readmission rates was collected.

Results: A total of 231 patients were subjected to Laparoscopic cholecystectomy in the ambulatory unit with overnight stay (time to discharge less than 24 hours). Three patients presented with intra-operative complications, which needed conversion to laparotomy, and four patients were admitted after surgery. The mean time for the procedure was 58 minutes and the mean time for recovery was $19 \mathrm{~h} 19$ minutes. Postoperative morbidity was $7.8 \%$ with 2 nonscheduled admission having occurred.

Discussion: One of the controversies regarding laparoscopic cholecystectomy as an outpatient procedure is the need for overnight hospital surveillance. In our unit, we have started LC on an outpatient basis with an overnight stay. Using this protocol, over the past two years, we have operated 231 patients and our results show that this is a completely safe technique.

Conclusions: Data from the study suggests that LC is a safe technique when performed in ambulatory practice, having similar results to other european surgical centers.

Keywords: Ambulatory Surgical Procedures; Cholecystectomy, Laparoscopic; Length of Stay; Morbidity.

\section{INTRODUÇÃO}

A colecistectomia laparoscópica (CL) tornou-se o gold standard para o tratamento da patologia vesicular benigna após diversos estudos terem mostrado uma morbilidade semelhante à abordagem por via laparotómica, mas com diminuição do tempo de permanência em meio hospitalar e do tempo de recuperação. ${ }^{1,2}$

A legislação portuguesa define a cirurgia de ambulatório como uma 'intervenção cirúrgica programada, realizada sob anestesia geral, loco-regional ou local que, embora ha- bitualmente efectuada em regime de internamento, pode ser realizada em instalações próprias, com segurança e de acordo com as actuais legis artis, em regime de admissão e alta no período inferior a 24 horas' $^{3}$

Com o reconhecimento de benefícios económicos e sociais associados à cirurgia em regime de ambulatório, diversos procedimentos passaram a ser realizados sem a necessidade de internamento. Uma revisão com meta-análise mostrou que a CL realizada em regime de ambulatório

1. Serviço de Cirurgia Geral. Hospital de Braga. Braga. Portugal.

Recebido: 28 de Novembro de 2012 - Aceite: 30 de Abril de 2013 | Copyright $\odot$ Ordem dos Médicos 2013 
é segura e pode ser realizada na maioria dos doentes. ${ }^{4}$

A Unidade de Cirurgia de Ambulatório do Hospital de Braga iniciou em 2010 uma recolha sistemática de diversos indicadores de qualidade e segurança dos procedimentos realizados em cirurgia de ambulatório.

O objectivo do presente trabalho é analisar esses indicadores de qualidade e segurança e comparar os resultados obtidos com os de outros centros europeus que realizam CL em regime de ambulatório.

\section{MATERIAL E MÉTODOS}

Foi realizado um estudo observacional prospectivo de doentes submetidos a CL em regime de Cirurgia de Ambulatório no Hospital de Braga entre 1 de Janeiro de 2010 e 29 de Fevereiro de 2012 pela Unidade Funcional de Cirurgia de Ambulatório de Cirurgia Geral do Hospital de Braga.

Foram seleccionados para a realização de CL em regime de ambulatório todos os doentes com litíase vesicular sintomática, com co-morbilidades compensadas (até ASA III compensados) e sem aparente necessidade de exploração da via biliar intra-operatoriamente. Não foram excluídos os doentes que apresentaram previamente episódios de colecistite ou pancreatite litiásica, nem doentes com cirurgias abdominais prévias, mesmo nos quadrantes superiores.

Os doentes seleccionados para o regime de Cirurgia de Ambulatório cumprem os critérios definidos pelo Departamento de Cirurgia de Ambulatório no Hospital de Braga:

a) Idade superior a cinco anos

b) Ser acompanhado de um adulto responsável, que possa estar com o doente desde a alta do Hospital até pelo menos 24 horas após a cirurgia

c) Ter transporte assegurado em veículo automóvel

d) Ter telefone/telemóvel para contactar a Unidade de

Cirurgia de Ambulatório em caso de necessidade

e) Ter condições logísticas adequadas no domicílio

f) Tempo entre o domicílio do doente e o Hospital de Braga não superior a 60 minutos

g) Duração previsível da intervenção não superior a 120 minutos

Os doentes foram avaliados pré-operatoriamente pelo Cirurgião, pelo Anestesiologista e pela Enfermeira da Unidade de Cirurgia de Ambulatório, tendo sido entregue um folheto informativo, onde é explicado o que é a Cirurgia de Ambulatório, quais os procedimentos a ter na véspera e no dia da intervenção e como proceder no caso de surgirem complicações.

Todos os doentes pernoitaram no recobro da Unidade de Cirurgia de Ambulatório, excepto dois doentes que tiveram alta no mesmo dia da cirurgia (em média oito horas após a cirurgia). No dia seguinte à cirurgia os doentes foram reavaliados pelo cirurgião responsável. No momento da alta hospitalar, quer o doente quer o seu acompanhante receberam informações relativas aos cuidados a ter no período pós-alta (cuidados de penso, informação clínica para o médico assistente, indicações para retirar os pontos, vigilância de sinais de alarme e necessidade de repouso relati- vo e dieta adequados à intervenção cirúrgica).

No fim de cada cirurgia, a Unidade Funcional de Cirurgia de Ambulatório de Cirurgia Geral recolheu sistematicamente vários parâmetros do doente operado e do respectivo procedimento cirúrgico, tendo essa informação sido inserida numa base de dados, utilizando o software Microsoft Office Excel ${ }^{\circledR}$. Os parâmeros recolhidos foram:

- Idade

- Sexo

- Classificação do risco da American Society of Anesthesiology (ASA)

- Categoria do cirurgião principal (interno vs especialista)

- Complicações operatórias

- Taxa de conversão

- Tempo cirúrgico

- Tempo recobro

Todos os doentes foram convocados para uma consulta pós-operatória de Cirurgia Geral com o Cirurgião responsável (entre 30 a 60 dias pós-cirurgia). Nesta consulta são registados os seguintes parâmetros:

- Morbilidade

- Taxa alta para o internamento

- Taxa readmissão

\section{Procedimento Cirúrgico}

Todos os doentes receberam profilaticamente $2 \mathrm{gr}$ de cefazolina, antes da incisão cirúrgica. A maioria dos doentes foi submetida a CL com a introdução de três trocares (um trocar de $5 \mathrm{~mm}$ e dois trocares de $10 \mathrm{~mm}$ ) e no final do procedimento o local de introdução dos trocares foi infiltrado com uma mistura anestésica que continha $10 \mathrm{~mL}$ lidocaína a $2 \%$ e $10 \mathrm{~mL}$ de ropivacaína $7,5 \%$ para controlo da dor incisional pós-operatória.

\section{Outcome e análise estatística}

Os dados usados neste estudo foram retirados da base de dados da Unidade de Cirurgia de Ambulatório e dos registos da consulta pós-operatória. Foi feita uma análise descritiva dos dados através do software Microsoft Office Excel $^{\circledR}$.

\section{RESULTADOS}

No período analisado, foram submetidos a $C L$ em regime de ambulatório pela UFCA de Cirurgia Geral do Hospital de Braga duzentos e trinta e um doentes, em média, nove doentes por mês.

A maioria dos doentes era do sexo feminino $(74,5 \%)$ com média de idades de 49,8 anos (mínimo de 19 anos e máximo de 84 anos). Segundo a classificação ASA 47,3\% estavam no estádio I, $51,1 \%$ no estádio II e 1,6\% no estádio III. Os doentes apresentavam Índice de Massa Corporal (IMC) médio de $27,7 \mathrm{~kg} / \mathrm{m}^{2}$ (mínimo de $17,1 \mathrm{~kg} / \mathrm{m}^{2}$ e máximo de $46,2 \mathrm{~kg} / \mathrm{m}^{2}$ )

O tempo médio de cirurgia foi de 58 minutos (mínimo de 29 minutos e máximo de 124 minutos). Após a cirurgia, os doentes estiveram no recobro da Unidade de Cirurgia de 
Ambulatório em média 19h19m (mínimo de 7h40m e máximo de 23h23m).

Ocorreu conversão para laparotomia em três casos $(1,3 \%)$ :

- um doente com antecedentes de cirurgia abdominal superior no qual ocorreu perfuração intestinal com a entrada do primeiro trocar a nível umbilical;

- um doente com hipotensão refratária após introdução do primeiro trocar umbilical (por suspeita de lesão vascular foi realizada conversão para laparotomia, tendo-se verificado não haver nenhuma lesão vascular nem outra complicação cirúrgica);

- um doente por dificuldade na dissecção e identificação das estruturas do triângulo de Callot.

A taxa de complicações cirúrgicas observadas durante a cirurgia foi de $0,4 \%$ (um doente com perfuração intestinal).

A admissão hospitalar não prevista (sem alta após $24 \mathrm{~h}$ da cirurgia) ocorreu em quatro doentes $(1,7 \%)$ :

- dois dos três doentes que foram convertidos para laparotomia (o doente que foi convertido por suspeita de lesão vascular teve alta para o domicílio);

- um doente por dor abdominal intensa na altura da avaliação pelo cirurgião tendo tido alta hospitalar dois dias após a cirurgia, sem evidência de complicação cirúrgica e/ou anestésica

- um doente por falta de vaga no recobro da Unidade de Cirurgia de Ambulatório.

A morbilidade aos 30 dias, avaliada segundo a classificação das complicações cirúrgicas de Dindo ecolaboradores $^{5}$ (Tabela 1), foi de $7,8 \%$, sendo na sua grande maioria de grau $\mathrm{I}$.

A taxa de reingresso hospitalar (vindas ao Serviço de Urgência) foi de $6,5 \%$ e a de readmissão hospitalar (internamentos não previstos após alta) foi de $0,9 \%$. Os doentes readmitidos encontram-se descritos na Tabela 2 . O doente que apresentou um hematoma, com necessidade de internamento e drenagem percutânea, tratava-se de um doente hipocoagulado com varfine por fibrilação auricular, cuja hipocoagulação oral foi suspensa e reintroduzida de acordo com as indicações do Serviço de Imuno-Hemoterapia.

\section{DISCUSSÃO}

Uma das discussões que existe em torno da CL em regime de ambulatório é a necessidade de vigilância hospitalar durante a primeira noite após a cirurgia. Uma meta-análise recente que pretendeu avaliar as vantagens e desvantagens da realização da CL com e sem pernoita hospitalar concluiu que a CL pode ser feita de forma segura sem necessidade de pernoita, mas alerta para a falta de estudos clínicos que comparem em termos de ansiedade e satisfação dos doentes submetidos a CL com e sem pernoita hospitalar. ${ }^{4}$

Tabela 1 - Classificação da morbilidade registada nos 30 dias pós-operatórios.

\begin{tabular}{|c|c|c|}
\hline Grau & $\mathrm{N}^{\circ}$ & Descrição \\
\hline I & $\begin{array}{l}6 \\
4 \\
2 \\
1 \\
1 \\
1\end{array}$ & $\begin{array}{ll}\text { Vinda ao } & \text { SU por: } \\
\text { - } & \text { Dor » reforço analgesia } \\
\text { - Infecção local trocar» com necessidade drenagem } \\
\text { - } \quad \text { Inflamação local trocar » sem necessidade drenagem } \\
\text { - } \quad \text { Seroma local trocar » sem necessidade drenagem } \\
\text { - } \quad \text { Derma membro interior » Eco-doppler normal » Alta com AINES + HBPM } \\
\text { - }\end{array}$ \\
\hline II & & \\
\hline IIla & 1 & $\begin{array}{l}\text { Biloma (vinda ao SU } 13 \text { dias após a cirurgia)» internamento + drenagem percutânea } \\
\text { Hematoma (vinda ao SU } 10 \text { dias após a cirurgia)» internamento + drenagem percutânea }\end{array}$ \\
\hline IIIb & 1 & Hérnia umbilical incisional (detectada na consulta pós operatória)» herniorrafia electiva \\
\hline IVa & & \\
\hline IVb & & \\
\hline $\mathbf{v}$ & & \\
\hline
\end{tabular}

Tabela 2 - Características dos doentes readmitidos.

\begin{tabular}{|c|c|c|c|c|c|c|c|c|}
\hline Doente & Idade & Sexo & ASA & $\begin{array}{l}\text { Duração } \\
\text { cirurgia }\end{array}$ & $\begin{array}{c}\text { Tempo } \\
\text { admissão SU } \\
\text { (dias) }\end{array}$ & Diagnóstico & Tratamento & $\begin{array}{c}\text { Tempo } \\
\text { internamento } \\
\text { (dias) }\end{array}$ \\
\hline 1 & 73 & Fem & 2 & $54 m$ & 13 & Biloma & $\begin{array}{l}\text { Drenagem } \\
\text { percutânea }\end{array}$ & 5 \\
\hline 2 & 78 & Mas & 2 & $112 m$ & 10 & Hematoma & $\begin{array}{l}\text { Drenagem } \\
\text { percutânea }\end{array}$ & 10 \\
\hline
\end{tabular}


Tabela 3 - Comparação com outros centros europeus.

\begin{tabular}{|c|c|c|c|c|c|}
\hline & $\begin{array}{c}\text { Braga } \\
\text { (Portugal) } \\
2012\end{array}$ & $\begin{array}{c}\text { Thomsen, } C^{6} \\
\text { (Dinamarca) } \\
2011\end{array}$ & $\begin{array}{c}\text { Marinis, } A^{7} \\
\text { (Grécia) } \\
2010^{b)}\end{array}$ & $\begin{array}{c}\text { Pérez, M }{ }^{8} \\
\text { (Espanha) } \\
\left.2010^{c}\right)\end{array}$ & $\begin{array}{c}\text { Briggs, } C^{9} \\
\text { (UK) } \\
\left.2009^{e}\right)\end{array}$ \\
\hline $\mathbf{N}^{\circ}$ doentes & 231 & 201 & 110 & 110 & 106 \\
\hline Idade média (anos) & 49,8 & 48,6 & 40,6 & 51,0 & 42,0 \\
\hline ASA I:II:III (\%) & $47: 51: 2$ & - & 89:11:0 & - & 43:63:0 \\
\hline Sexo Masculino & $26 \%$ & $30,3 \%$ & $29 \%$ & $26,4 \%$ & $13,2 \%$ \\
\hline Cirurgião Interno & $33,7 \%$ & $31,4 \%$ & $81,8 \%$ & - & - \\
\hline Tempo cirúrgico & $58 m$ & - & $43,8 m$ & - & $62 m$ \\
\hline Tempo recobro & $19 \mathrm{~h} 11 \mathrm{~m}$ & - & - & - & $7 \mathrm{~h} 07 \mathrm{~m}$ \\
\hline Taxa conversão & $1,3 \%$ & $3,0 \%$ & $0 \%$ & $0,9 \%$ & $1,9 \%$ \\
\hline Complicações operatórias & $0,4 \%$ & $2,0 \%$ & - & - & - \\
\hline Taxa alta internamento & $1,7 \%$ & - & - & $(9 \%>1 \mathrm{dia})$ & - \\
\hline Morbilidade & $7,8 \%$ & $4,5 \%$ a) & $15,5 \%$ & $6,4 \%^{\mathrm{d})}$ & - \\
\hline Taxa readmissão & $0,9 \%$ & - & $1,8 \%$ & $0,9 \%$ & $1,9 \%$ \\
\hline
\end{tabular}

a) Não descreve nenhuma ida ao médico por dor.

b) Exclui colecistites prévias, ASA $\geq 11$ e IMC $\geq 30 \mathrm{~kg} / \mathrm{m} 2$.

c) Exclui colecistites prévias, $A S A \geq I I I$ e ausência anti-coagulantes ou anti-agregantes.

d) Não considera a ida ao SU por dor mal controlada como morbilidade.

e) Exclui ASA $\geq I I$, IMC $\geq 38 \mathrm{~kg}$ / m2, cirurgia abdominal prévia e doença biliar complicada.

Na nossa unidade, e de acordo com a nossa legislação que permite a realização de cirurgia de ambulatório com pernoita (ao contrário de outros países europeus como a Espanha, França e Inglaterra nos quais a alta do ambulatório tem de ser no mesmo dia que a cirurgia), iniciamos a realização da $C L$ em regime de ambulatório com pernoita hospitalar. Os objectivos foram de iniciar com segurança a realização de um procedimento cirúrgico intra-abdominal em regime de ambulatório com a monitorização durante a primeira noite por uma equipa de enfermagem treinada e de dar segurança ao doente de que estava acompanhado por profissionais de saúde durante as primeiras 24 horas.

Os resultados de mais de dois anos e 231 doentes operados a CL mostram que é uma técnica perfeitamente segura em regime de ambulatório: taxa de conversão para laparotomia 1,3\%, complicações operatórias $0,4 \%$, morbilidade aos 30 dias 7,8\% (maioria minor), e taxa de readmissão hospitalar de $0,9 \%$.

Comparando com estudos de outros centros europeus, os nossos resultados são bastante similares (Tabela 3). Um dos indicadores que é difícil de comparar é a taxa de alta para internamento, pois nos outros centros é mais alta por não haver a possibilidade de pernoita hospitalar.

\section{REFERÊNCIAS}

1. Soper NJ, Stockmann PT, Dunnegan DL, Ashley SW. Laparoscopic cholecystectomy. The new 'gold standard'? Arch Surg. 1992;127:917-21.

2. Keus F, de Jong JA, Gooszen HG, van Laarhoven CJ. Laparoscopic versus open cholecystectomy for patients with symptomatic cholecystolithiasis. Cochrane Database Syst Rev. 2006:CD006231.

3. Diário da República. $1 .^{a}$ série - N. ${ }^{\circ} 21$ - Portaria n 132/2009.

4. Gurusamy K, Junnarkar S, Farouk M, Davidson BR. Meta-analysis of randomized controlled trials on the safety and effectiveness of day-case laparoscopic cholecystectomy. Br J Surg. 2008;95:161-8.

\section{CONCLUSÕES}

Os resultados da nossa unidade mostram que a CL é uma técnica segura em regime de ambulatório, com resultados similares a outros centros europeus.

A realização de $C L$ em regime de ambulatório sem pernoita pode ser uma evolução no que actualmente se pratica no nosso centro cirúrgico, tendo-se contudo de ponderar se a abdicação da pernoita não aumentará a ansiedade e afectará a satisfação dos doentes, diminuindo desta forma o grau de qualidade deste procedimento cirúrgico em cirurgia de ambulatório.

\section{CONFLITO DE INTERESSES}

Os autores declaram que não houve conflito de interesses na realização deste trabalho.

\section{FONTES DE FINANCIAMENTO}

Os autores declaram a inexistência de fontes de financiamento externos. Resultados do estudo apresentados no I Congresso Ibérico de Cirurgia Ambulatória / VII Congresso Nacional de Cirurgia Ambulatória, Braga - Maio de 2012.

5. Dindo D, Demartines N, Clavien PA. Classification of surgical complications: a new proposal with evaluation in a cohort of 6336 patients and results of a survey. Ann Surg. 2004;240:205-13.

6. Thomsena C, Bordingb C, Rasmussenc S. Day case laparoscopic cholecystectomy in a centre with more than 10 years experience in ambulatory surgery: indications, complications, length of stay, and readmissions. Ambulatory Surg. 2011;17.

7. Marinis A, Stamatakis E, Tsaroucha A, Dafnios N, Anastasopoulos G, Polymeneas G, et al. Safety and effectiveness of outpatient laparoscop- 
ic cholecystectomy in a teaching hospital: a prospective study of 110 consecutive patients. BMC Res Notes. 2010;3:207.

8. Lezana Perez MA, Carreno Villarreal G, Fresnedo Perez R, Lora Cumplido P, Padin Alvarez H, Alvarez Obregon R. Colecistectomía laparoscópica en régimen de cirugía mayor ambulatoria en un hospital comarcal: resultados iniciales de una serie de 110 casos. Cir Esp.
2010;87:288-92.

9. Briggs CD, Irving GB, Mann CD, Cresswell A, Englert L, Peterson M, et al. Introduction of a day-case laparoscopic cholecystectomy service in the UK: a critical analysis of factors influencing same-day discharge and contact with primary care providers. Ann R Coll Surg Engl. 2009;91:58390 . 